\title{
Unformed lateralization of the brain hemispheres regarded as a neuropsychological feature of primary school children
}

\author{
Inna Lukyanova ${ }^{1,}$, Evgeniy Sigida ${ }^{1}$, Svetlana Utenkova $^{1}$, Svetlana Dmitrieva ${ }^{1}$, Maria \\ Chibrikova $^{1}$ \\ ${ }^{1}$ Moscow Region State University, 10A, Radio str, 105005, Moscow, Russia
}

\begin{abstract}
The purpose of the study is to identify the neuropsychological features of primary school children (unformed lateralization of the brain hemispheres) that substantiate the necessity for changes in the organization of their learning process. The data of the research demonstrated the need to classify children of primary school age in accordance with the characteristics of their perception and the type of thinking. In the system of interactions between the brain hemispheres, signal systems and the type of thoughts, 7-8 year old pupils displayed the priority of reflecting the world through the first-signal system, stimulating the dominance of the activity of the right hemisphere, which determines the dominance of visual thinking. Children who are from 9 to 10 years old are characterized by the reflection through a second-signal system, a shift of the brain lateralization to the left hemisphere, and the development of abstract thinking. The results described above suggest that only at the age of 9-10, modern primary school children are ready to learn within the existing educational system; 7-8-year-old children are much worse at perceiving information transmitted through the words and are not ready for a learning pattern based on the use of abstract thinking; forced switching of children of this group from the right-hemisphere lateralization of the brain to the lefthemisphere leads to a state of hemispheric dysfunction and, as a result, to neurotic disorders. The education of primary school children must be approached in a differentiated way. The education system of 7-8 year old pupils, whose thinking needs to complete the development of visual thinking (not to leave the process incomplete) and to proceed to the formation of a second-signal system with the dominance of the left hemisphere, which determines the transition to abstract thinking, needs to be rebuilt by excluding the factors that traumatize neuro-physiological structures from it.
\end{abstract}

\section{Introduction}

At the present time, individual characteristics of perception and, as a result, the child's thought associated with hemispheric asymmetry are rarely taken into account in the

*Corresponding author: 7828262@inbox.ru 
education system. Despite the fact that the training practice of any specialist who would have to work with children involve the formation of competencies aimed to take into consideration the peculiarities of the child's development [7], the education process still does not focus on the need to take into account such an important feature of children as the lateralization of the hemispheres. In rare cases, the left- and right-handedness are considered $[1,2]$. However, paying attention to the special needs of left-handed people in a relatively right-handed world is far from everything that needs to be noted $[3,9]$. Timely diagnostics of the lateralization of brain function includes the study of the correlation of signal systems and allows to determine the type of thought that dominates at a particular stage of ontogenesis. This provides the basis for the organization of adaptive developmental (or corrective developmental, in special cases) environment [6].

The purpose of the study is to identify the neuropsychophysiological characteristics of primary school children, that substantiate the need for changes in the organization of the learning process. Within the stated age, the important changes occur in the development of higher mental functions. There is a serious reorganization - a transition from the visual type of thinking to the abstract thought. And this transition does not come easily for all children. In the modern education system, it is not considered that a child of this age might not be ready to operate with abstract categories. As a result, these children could follow two paths. The first could be described by the fact that, despite the obvious normativity of a child with a special neurophysiological mindset, the education system does not adapt to the child's needs, and the child is forced to break his/her own natural abilities in order to switch to generally accepted education scheme, which, in all cases, leads to neurotic disorders, arising due to the emergence of hemisphere dysfunction [8]. The second way is based on the the educational system's response - a child who has a weaker type of higher nervous activity and who is not able to break his/her mental nature is classified to a group of children with developmental delay. Thereafter, the system, additionally making efforts, forcibly transfers the child to the first path or consider him/her to have intellectual disabilities, due to the inability to operate with abstract categories.

In primary school age, thinking is closely related to the development of speech [4]. And this is justified by the transition to abstract thinking. As noted by Vygotsky L.S., "entry into consciousness is possible by changing the level of generalizations (from the semantic perspective) through speech activity", contributing to the emergence of the most important psychological formations: the development of verbal (abstract) thinking, mental ability, concept thinking [5].

Visual thinking in actions, images is a complex process, which reflects the results of sensory perception of the surroundings under the influence of the child's internal attitudes, awareness of the external environment with the use of visual internal images. Normal mental development is primarily grounded in an imaginative perception of the surroundings and the ability to create mental images and operate with them which creates the basis for further development of the child's intelligence.Visual thinking is carried out with the direct participation of the environment even before the development of speech structures and becomes a necessary condition for the formation of verbal reasoning. Logic, analysis, generalization, language acquisition device could not be formed prior to the development of such abilities as perception of a sensory image, recognition of the shape of an object, orientation in space and other abilities that are developed with the involvement of visual thinking. A child needs to be provided with the opportunity to complete the development within the framework of this type of thinking that precedes the abstract thought, as well as to receive the maximum life experience possible at this development stage. Every mental process that has begun must necessarily be completed. Natural development must not be disrupted. The abortive course of development of visual thinking and untimely and abrupt transition to a new stage in the form of a forced formation of abstract thought, negatively 
affects the overall development. In contrast, the formed, developed, visual thinking becomes a launch pad for the further mental development. The group of children of primary school age is heterogeneous from the perspective of the positions discussed above - a significant part of children is at risk. It is necessary to carefully study the distinctive features of development of their thinking, taking into account the lateralization of the brain function and the correlation between signal systems. The issue is still insufficiently developed and requires the extensive research. Therefore, the purpose of the study is to identify the characteristics of thinking of primary school children, taking into account the lateralization of the brain function and the correlation between signal systems.

\section{Methods}

1. Identification of the dominant hemisphere (sensorimotor asymmetry) and the individual asymmetry of the body sides.

The selection of appropriate methodologies was carried out on the basis of the following parameters:

- motor skills (conditioned motor analyzer) - an important indicator of symmetry, which could not be ignored, however, it is dependent on the features of ontogenesis (possible asynchronies, developmental anomalies, etc.) and external conditions (injuries, conditions of upbringing, etc.), and, therefore, could not be accepted as a leading parameter;

- visual, auditory and somato-sensory perception data are recognized as fundamental in determination of brain laterization.

The evaluation of the perception results of the asymmetry of each analyzer (for each task test) was conducted in conditional numbers and as a percentage. The "motor skills" asymmetry (MA) was determined by the formula:

$$
\mathrm{MA}=\mathrm{A}+\mathrm{BA}
$$

where MA - motor asymmetry, A - asymmetry, BA - body asymmetry.

The "sensory" asymmetry was calculated by the formula:

$$
\mathrm{SA}=\mathrm{AVP}+\mathrm{AAP}+\mathrm{ASSP}
$$

where SA - sensory asymmetry, AVP - asymmetry of visual perception, AAP - asymmetry of auditory perception, ASSP - asymmetry of somato-sensory perception.

The general function asymmetry was determined by the formula:

$$
\mathrm{GFA}=\mathrm{MA}+\mathrm{SA},
$$

where GFA - general function asymmetry, MA - motor asymmetry, SA - sensory asymmetry.

2. The methodology of Klimov E.A. was applied as the basis for studying the correlation between signal systems in different age groups of children [10].

\section{Results}

The study involved 108 primary school children who were classified into two groups. The idea to group children emerged due to the fact that from the neuropsychological perspective 7-8-year-old children are extremely close to older preschoolers, as the functional organization of their brain is still in the process of re-orientation. Therefore, 7-8-year-old children were identified as a group of beginner primary school students (BPSS). The second group that involved 9-10-year-old children was defined as a group of non-beginner primary school students (NBPSS). 
At the first stage, both groups were examined to identify the dominant hemisphere and an individual asymmetry of the sides of the body.

The data compiled on the basis of indicators of sensory-motor asymmetry of the body of children of primary school age are presented in Table 1.

Table 1. Sensory-motor indicators of body asymmetry.

\begin{tabular}{|c|l|l|l|l|l|}
\hline Group & \multicolumn{1}{|c|}{ Analyzer } & \multicolumn{2}{c|}{ Left side of the body } & \multicolumn{2}{c|}{ Right side of the body } \\
\hline \multirow{5}{*}{ BPSS } & $\begin{array}{l}\text { Conditioned motor } \\
\text { analyzer }\end{array}$ & 97 & $28.4 \%$ & 49 & $23.7 \%$ \\
\cline { 2 - 6 } & Visual perception & 77 & $22.6 \%$ & 36 & $17.4 \%$ \\
\cline { 2 - 6 } & Auditory perception & 94 & $27.6 \%$ & 50 & $24.2 \%$ \\
\cline { 2 - 6 } & Somato-sensory perception & 73 & $21.4 \%$ & 72 & $34.7 \%$ \\
\cline { 2 - 6 } Total: & 341 & & 207 & \\
\hline \multirow{5}{*}{ NBPSS } & $\begin{array}{l}\text { Conditioned motor } \\
\text { analyzer }\end{array}$ & 39 & $29.5 \%$ & 90 & $26.8 \%$ \\
\cline { 2 - 7 } & Visual perception & 26 & $19.8 \%$ & 74 & $22.0 \%$ \\
\cline { 2 - 6 } & Auditory perception & 28 & $21.2 \%$ & 86 & $25.6 \%$ \\
\cline { 2 - 6 } & Somato-sensory perception & 39 & $29.5 \%$ & 86 & $25.6 \%$ \\
\cline { 2 - 6 } & \multicolumn{2}{|c|}{ Total: } & 132 & 336 & \\
\hline
\end{tabular}

The determination of the dominant side of the body allows to identify the leading hemisphere. In this case, the Bogen's rule is applied, according to which the dominant hemisphere is the one that is opposite to the leading side of the body. Thus, the data presented in the table within the definition of hemispheric asymmetry correspondingly provide the opposite results.

As depicted in Table 1, the majority of children of the BPSS group possess the dominating sensory systems of the left side of the body; therefore, it could be stated that this is a case of left-sided body lateralization with the dominance of the right hemisphere.

In the examined children of the NBPSS group, the predominance of sensory systems of the right half of the body was revealed, which indicates the activation and the beginning dominance of the left hemisphere.

Further studies allowed to study the characteristics of the interaction of children of the selected groups with the environment, through the correlation between the first- and second-signal systems.

The results of the research conducted in two age groups are presented in Figure 1.

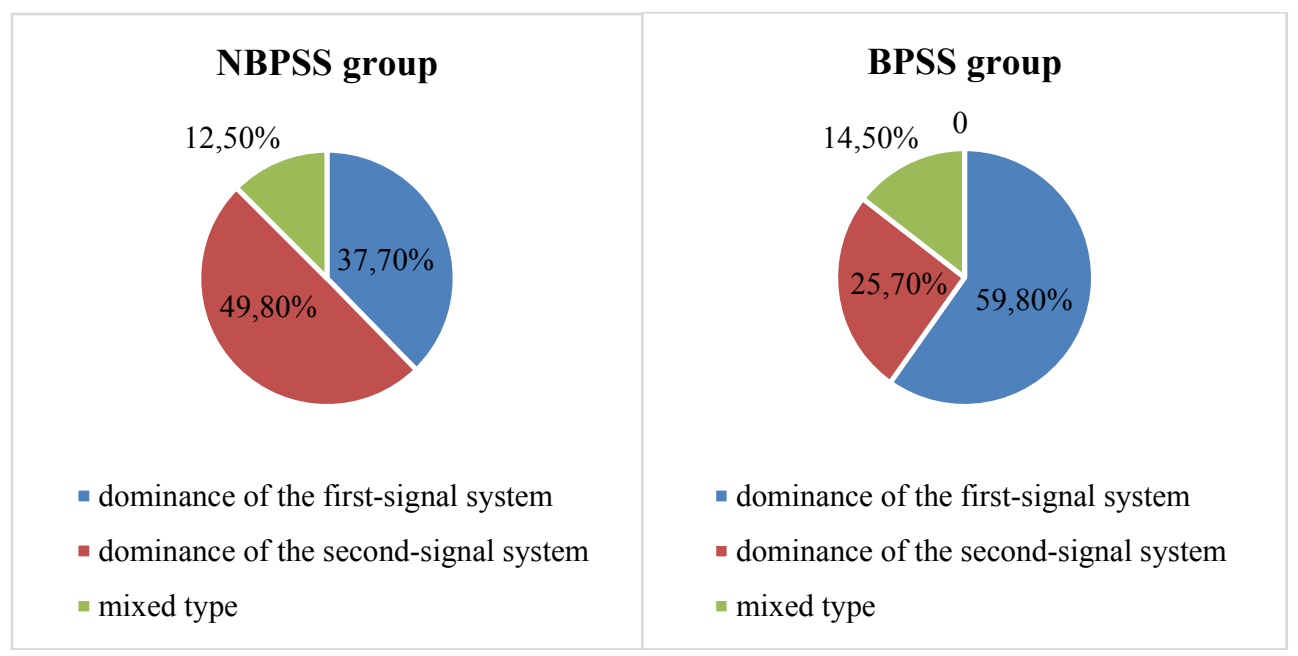

Fig. 1. Diagrams of the correlation between the signal systems in the studied age groups. 
The analysis of the diagrams demonstrates that in the BPSS group, the first-signal system is manifested in $59.8 \%$ of cases, the second-signal system is revealed in $25.7 \%$, and a mixed type was detected in $14.5 \%$ of children.

The results in the NBPSS group are opposite: the percentage of indicators of the dominance of the first-signal system is significantly reduced and observed in $37.7 \%$ of respondents, while the percentage of children with a sufficient level of development of the second-signal system is abundantly increased (49.8\%). A mixed type was found in $12.5 \%$ of respondents.

\section{Discussion}

Diagnostic results of children of the BPSS group display that at the initial stage of education at school, a child's brain, both in general and in most cases, maintains right-brain lateralization, which entails neuropsychological features - the child's interaction with the external environment at the stage is provided by sensory perception, intuitive activity and the importance of direct interaction with the environment and society. Thinking is associated with reality, direct observation, and holistic perception. The incoming information and its identification is carried out in accordance with the existing and emerging images. The perception of verbal information is difficult, the development of abstract thinking of most children is delayed.

At the age of 9-10, a child begins to feel the need to process an increasing flow of information, the communication becomes complicated, there is a necessity for abstraction from the external influence, and the change of the dominant functions of the hemispheres occurs. The left-brain lateralization is shaped, and thereafter emerged.The qualities of the right hemisphere gradually stop their external manifestation. Therefore, abstract thinking begins to dominate.

The change of forms of thinking, as well as the heterogeneity in the context of functions of the left and right hemispheres are the neuropsychological basis of the age crisis that leads to a special psychological state which is the right hemisphere dysfunction (RHD). Hemispheric dysfunction could be manifested by a child's state of frustration, depression with signs of irritability, with frequent mood changes, impairment in the ability to perceive non-standard information, and to maintain the emotionality of speech [8]. This condition is especially critical for the children who have not fully passed through the stage of visual thinking and who, with the dominant right hemisphere, are required to demonstrate indicators peculiar to abstract thinking. First of all, it significantly reduces the motivation for cognitive activity and, thereafter, does not allow the proper formation of the new functions - most notably, the perception of a word as a "signal of signals".

Generalized development indicators of primary school children of groups of beginner and non-beginner primary school students are displayed in Table 2.

Table 2. The correlation between the dominant hemisphere, signal systems and the type of thinking of primary school students.

\begin{tabular}{|l|l|l|l|}
\hline \multicolumn{1}{|c|}{ indicator } & \multicolumn{1}{c|}{$\begin{array}{c}\text { Dominant } \\
\text { hemisphere }\end{array}$} & Dominant signal system & \multicolumn{1}{|c|}{ Type of thinking } \\
\hline BPSS & Right hemisphere & First-signal system & Visual thinking \\
\hline NBPSS & Left hemisphere & Second-signal system & Abstract thinking \\
\hline
\end{tabular}

A combined study of the results in two groups reveal that children of the BPSS group are characterized by a clear dominance of the first-signal system in the interaction with the environment. This explains the phenomenon of a better, in comparison with the indicators of the NBPSS group, orientation in space and visual memory span, providing the object 
perception and picture thinking. The dominance of the first-signal system also points out the dominant role of the right hemisphere of this age category within the interaction with the environment, providing a better perception of physical characteristics of stimuli (smell, color, visual sensation).

In the BPSS group, the number of children with the dominant second-signal system significantly increases and prevails over the number of children with a transitional type and a remaining dominance of the first-signal system. The second signal-system is necessary for the perception of verbal and written material and the development of logical thinking. In the organization of the second-signal system, the left hemisphere is more involved and activated, providing recognition of the identity between stimuli and the verbal indicators. Children of the older age group demonstrate a slowdown in the development of such abilities as the integrity of perception, the level of imagination decreases - they fantasize less and define the thoughts more clearly.

The apparent connection between the indicators of the correlation between the dominant hemisphere, signal systems and a type of thinking in primary school children is discovered. The development level of thinking and, as a consequence, its type, are significantly distinctive in different age groups. In the BPSS group, mental activity is carried out grounding in the interaction with the social environment on the basis of a wider range of sensory abilities, due to natural mental functions. The child easily responds to the stimuli that he/she sees, touches, feels, not fully acquiring the verbal information. Understanding is achieved by the recognition of the physical identity between the stimuli, and not by the word heard or seen. Children from the BPSS group often encounter difficulties when it is necessary to analyze and predict situations. Children with a dominance of object thinking, determined by the work of the first-signal system, as an older form of interaction, are in a wide range of their abilities - from giftedness to mental retardation and developmental delay, and in accordance with Pavlov's classification, could be classified into the artistic personality type. The mental development of these children occurs under conditions of dominance of the right hemisphere or, figuratively speaking, delayed lateralization of brain function. Children with a right-brain dominance display a special sensory-motor perception, and the application of pedagogical methods, taking into account the influence of the firstsignal system, allows to achieve better results in the education process. At this stage, a particular attention should be paid to the hand movements. Corrective development work should be directed from movement to thinking, and not vice versa.

The well-known aphorism of Sauniere I. should be mentioned where the attention is drawn to the importance of formation of visual thinking in primary school children: "when teaching the left hemisphere, only the left hemisphere is trained, however, while teaching the right hemisphere, the whole brain is trained".

Abstract thinking is a more specific stage of mental development, for which the abilities of visual thinking, such as the ability to operate with images in space, to compare received information, etc must be prepared in a timely manner. The underdevelopment of visual thinking negatively affects the mental development of a child, which gives reason to regard visual thinking as a special structural element of the mental ontogenesis of primary school children and as a necessary natural condition for the development of speech and requires a particular psychological-pedagogical organization of corrective development activities.

Unfortunately, in the special (defectologic) education practice the individual characteristics of a child associated with the interhemispheric asymmetry are rarely taken into account. The educational process has not been re-oriented to the need to pay attention to the lateralization of the brain hemispheres. It is not taken into consideration that excessive pressure within the context of verbal-logical thinking, in conditions of underdevelopment of visual thinking, leads to an impairment of normal mental ontogenesis, as well as to a breakdown of a child's neuropsychic status. Timely diagnosics of the 
lateralization of brain function, the identification of the correlation between signal systems and the definition of the dominating type of thought reveal the psychophysiological characteristics of children of primary school age, which allows to determine the patterns of their education and upbringing.

For the special education practice, it is important to consider the development of various types and forms of thinking in dependence to the characteristics of the child's interaction with the environment within the functioning of the signal systems. The correlation between the dominance of signal systems and the sequence of formation of the major types of thought allows to predict the development of mental activity, general adaptability to the learning conditions, potential performance in the main groups of subjects and, accordingly, to determine the form of organization of corrective development events.

\section{References}

1. M. Annett, Left, Right, Hand and Brain: The Right Shift Theory (London, Lawrence Erlbaum, 1985)

2. J.B. Hellige, In Hemispheric asymmetry: What's right and what's left (Harvard Press, 2001)

3. K. Hugdahl, Lateralization of cognitive processes in the brain. ActaPsychol (Amst) 105(2-3), 211 (2000)

4. D. Kimura, Canadian Journal of Psychology 15, 156 (1961)

5. L.S. Vygotsky, Sobr. soch.: v 6-ti t. T.5. Osnovy defektologii (M., Pedagogika, 1983)

6. I.E. Lukyanova, E.A. Sigida, S.N. Utenkova, Spetsial'noye obrazovaniye Yekaterinburg 2(46) (2017)

7. I.E. Lukyanova, E.A. Sigida, S.N. Utenkova, Detskaya i podrostkovaya reabilitatsiya 3(31), 54 (2017)

8. I.E. Lukyanova, E.A. Sigida, S.N. Utenkova, Special education 1(53) (2019)

9. I.E. Lukyanova, E.A. Sigida, S.N. Utenkova, Detskaya i podrostkovaya reabilitatsiya 2(34) (2018)

10. S.G. Rosenthal, T.V. Baltina, A.A. Eremeev, Psikhofiziologicheskiye metody issledovaniya psikhicheskikh funktsiy cheloveka: Uchebno-metodicheskoye posobiye (Kazan', 2015) 